\title{
Katzenpfoten bei Typ IV-Allergie auf P-Phenylendiamin nach Henna-Tattoo
}

\section{Type IV Allergic Reaction to P-Phenylendiamin Results in Catpaw Like Eczema Following Henna Tattoo}

Autoren

Institute
C. Stetter ${ }^{1}$, T. Plaza ${ }^{2}$, P. von den Driesch ${ }^{3}$

Hautarztpraxis für Dermatologie und Allergologie, Stuttgart

Hautarztpraxis für Dermatologie und Allergologie, Uster/Schweiz

Klinik für Dermatologie und Allergologie, Zentrum für Hautkrankheiten, Klinikum Stuttgart
Bibliografie

DOI http://dx.doi.org/ $10.1055 / \mathrm{s}-0029-1243904$

Online-Publikation: 8. 2. 2010

Akt Dermatol 2010; 36:

229-231 @ Georg Thieme

Verlag KG Stuttgart · New York ISSN 0340-2541

Korrespondenzadresse Dr. med. Christoph Stetter Hautarztpraxis für Dermatologie und Allergologie Felix-Dahn-Straße 40 70597 Stuttgart dielstetter@hotmail.de

\section{Zusammenfassung $\nabla$}

Wir berichten von einer jungen Patientin, bei der es 3-5 Tage nach Applikation eines Henna-Tattoos zu einem allergischen Kontaktekzem in Form von Katzenpfoten kam. Als Ursache der Reaktion konnten wir eine Typ IV-Allergie auf ParaPhenylendiamin nachweisen. Durch eine Lokal-

\section{Einleitung \\ $\nabla$}

Tätowierungen erfreuen sich einer zunehmenden Beliebtheit in Europa, obwohl das Bundesinstitut für Risikobewertung (BfR) bereits 2004 und erneut 2007 eine Warnung für permanente und temporäre Tätowierungen herausgegeben hat, da aus diesen Tätowierungen gesundheitliche Schädigungen infektiöser, allergischer und immunologischer Genese resultieren können [1]. Ungeachtet dessen trägt fast jeder 10. Bundesbürger eine Tätowierung. Am häufigsten treten sowohl bei permanenten als auch bei temporären Tattoos allergische Hautreaktionen auf. Die benötigten Tätowierfarben sind in der Regel nicht als Fertigprodukte verfügbar. $\mathrm{Zu}$ ihrer Herstellung werden bei Permanent-Tattoos pulverisierte Farbpigmente verwendet, die z.B. Metalle oder Azofarbstoffe beinhalten. Die Farbstoffe für Henna-Tattoos enthalten neben pflanzlichen Allergenen gelegentlich auch hochpotente Allergene wie das in Deutschland verbotene Para-Phenylendiamin (PPD) [2,3].

\section{Kasuistik \\ $\nabla$}

Anamnese

Unsere Patientin ließ sich vor einem Jahr im Kroatienurlaub erstmals im Leben ein Henna-Tattoo anfertigen. Es traten keinerlei Komplikationen auf, sodass sie sich bei einem späteren Urlaub in therapie mit einem Klasse III-Steroid kam es zu einer raschen Abheilung. PPD ist ein wichtiges Gruppenallergen gegen Para-Stoffe, in unserem Fall zeigte sich eine Kreuzreaktion auf para-Toluylendiamin. PPD stellt nach wie vor ein nicht $\mathrm{zu}$ unterschätzendes Kontaktallergen dar und ist sowohl im beruflichen als auch im privaten Bereich von erheblicher Relevanz.

Spanien erneut einer Henna-Tätowierung unterzog. Ungefähr 3-5 Tage später kam es zu einer heftigen Ekzemreaktion mit beginnender Depigmentierung der dunklen Tätowierung und Umwandlung in eine stark juckende, scharf begrenzte Ekzemreaktion. Nach Rückkehr aus dem Urlaub stellte sich die Patientin in unserer Praxis ( $\bullet$ Abb. 1) zur Abklärung der Ursache und Therapie vor.

Unter Würdigung der Anamnese bestand der Verdacht, dass ein Inhaltsstoff des Henna-Tattoos Ursache für die Beschwerden der Patientin sein könnte. Daher entschieden wir uns zur Epikutantestung der DKG-Standardreihe, DKG-Friseurstoffe und P-Phenylendiamin. Außerdem führten wir eine Pricktestung auf Inhalativa durch.

\section{Dermatologischer Befund}

Im Bereich des linken Unterbauches zeigen sich scharf begrenzte, erythematös-infiltrative, teils lichenifizierte Herde mit der Konfiguration von Katzenpfoten ( $\bullet$ Abb. 1).

\section{Allergologische Befunde Epikutantestung}

Die Epikutantestung wurde nach Standardverfahren durchgeführt [4]. Die Ablesung erfolgte nach 48 und 72 Stunden ( Tab. 1).

Zweifach positive Reaktionen (Erythem, Infiltrat, Papeln, Vesikel): p-Toluylendiamin und P-Phenylendiamin (PPD). 


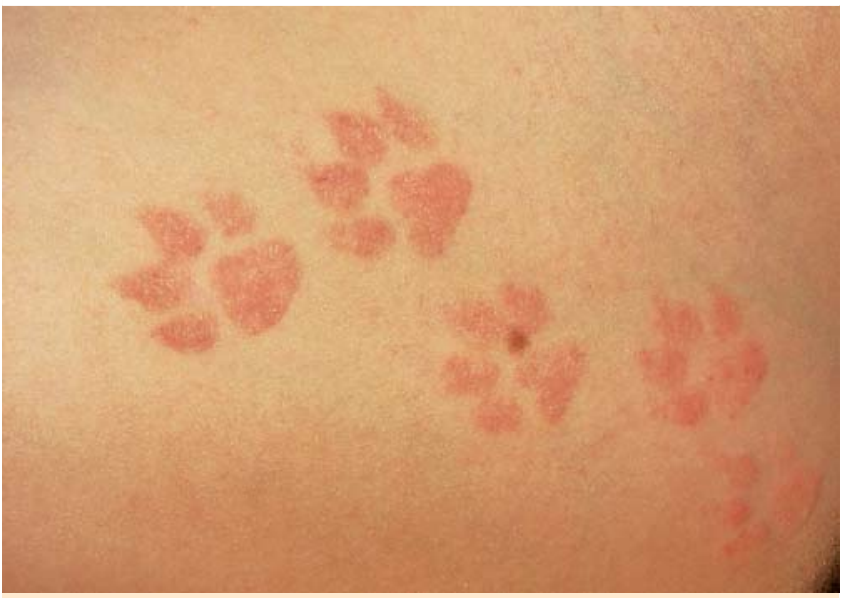

Abb. 1 Allergisches Kontaktekzem in Form der ursprünglichen HennaTätowierung als Katzenpfoten.

Einfach positive Reaktionen (Erythem, Infiltrat, diskrete Papeln): Monoethanolamin.

\section{Pricktestung}

Die Pricktestung auf Inhalativa wurde nach Standardverfahren durchgeführt [5]. Die Ablesung erfolgte nach 20 und 40 Minuten. Negativ: Bäume I (Frühblüher) und Bäume II (Mittelblüher), Gräser/Getreide, Kräutermischung, Ragweed, Dermatophagoides pteronyssinus, Dermatophagoides farinae, Acarus siro, Tyrophagus putrescentiae, Hundeepithelien, Katzenepithelien, Pilze I-Mischung (saisonal) und Pilze II-Mischung (ganzjährig), (alles Allergopharma); dreifach-positive Histaminkontrolle, negative $0,9 \%$ $\mathrm{NaCl}$-Kontrolle.

\section{Therapie und Verlauf}

Am Tag der Erstvorstellung erfolgte unabhängig von der allergologischen Abklärung eine hochpotente, rein lokale Steroidtherapie mit einem Klasse III-Steroid in Form von Betamethasonvalerat $0,1 \%$. Nach 14 Tagen Anwendungsdauer bestand nur noch eine flaue minimale Hyperpigmentierung, die sich unter blander Rückfettung und einem Klasse II-Steroid (Triamcinolonac. 0,1\%) dreimal pro Woche fast vollständig zurückbildete.

\section{Diskussion \\ $\nabla$}

Im vorliegenden Fall kam es nach der zweiten Henna-Tätowierung zu einer verzögerten ausgeprägten Ekzemreaktion. HennaTattoos stellen ein beliebtes Mitbringsel aus südlichen Ländern dar, wo Künstler sie auf Straßen und an Stränden günstig anbieten, ihr Ursprung geht jedoch bis ins Mittelalter zurück [10]. Bei Henna handelt es sich um ein dunkelgrünes Pulver aus den Blättern des Cyperstrauchs (Lawsonia inermis), der unter anderem in Indien, Afrika und auf Sri Lanka heimisch ist. Das Pulver wird mit Öl oder Wasser angerührt und verbleibt normalerweise für 2-6 Std. auf der Haut. Aufgrund der Anamnese bestand der Verdacht auf ein kontaktallergisches Geschehen auf einen Inhaltsstoff des Henna-Tattoos; in der durchgeführten Epikutantestung konnten wir eine Typ IV-Allergie auf P-Phenylendiamin (PPD) und p-Toluylendiamin nachweisen. P-Toluylendiamin ist wie P-Phenylendiamin (PPD) bekannt dafür, immer häufiger zur Farbintensivierung und Beschleunigung des Färbeeffektes den als Henna-Farbstoffen deklarierten Mischungen zur Anfertigung temporärer
Tab. 1 Ablesungsergebnis der Epikutantestung nach 48 und 72 Stunden.

\begin{tabular}{lll} 
Kontaktallergen & $\begin{array}{l}\text { Epikutantest- } \\
\text { reaktion } \mathbf{( 4 8 ~ h )}\end{array}$ & $\begin{array}{l}\text { Epikutantest- } \\
\text { reaktion }(\mathbf{7 2 ~ h )}\end{array}$ \\
$\begin{array}{l}\text { Paraphenylendiamin (PPD) } \\
(1 \% \text { in Vaseline })\end{array}$ & + & ++ \\
\hline $\begin{array}{l}\text { p-Toluylendiamin } \\
(1 \% \text { in Vaseline })\end{array}$ & + & ++ \\
$\begin{array}{l}\text { Monoethanolamin } \\
(1 \% \text { in Vaseline })\end{array}$ & $\varnothing$ & + \\
\hline
\end{tabular}

Tattoos beigemischt zu werden [6-8]. Im Gegensatz zu echten Tätowierungen dringen die Farblösungen bei temporären Tätowierungen (engl.: paint-on tattoo) nicht tief in die Haut ein. Eigentlich würde diese Form der Tätowierung dem Lebensmittelrecht und der Kosmetikaverordnung unterliegen, aber die Tätowierungsfarbstoffe und -lösungen stuft der Gesetzgeber nicht als Kosmetika ein und der Import wird nur oberflächlich kontrolliert [9]. Anstelle der traditionellen Henna-Farbe, die erst nach Stunden ihren vollen Effekt erzielen würde, kommt es durch die Beimischung von PPD zu tiefschwarzen Farbtönen, die mit größerer Präzision aufgetragen werden können, parallel lässt sich der Vorgang der Tätowierung auf wenige Minuten Anwendungsdauer beschleunigen [7]. Eine Untersuchung der Inhaltsstoffe von Henna-Tattoo-Mixturen ergab, dass PPD in einer Konzentration bis $15,7 \%$ nachgewiesen werden konnte $[6,9]$. Ferner ergab eine andere Studie, dass diese zu einem hohen Grad mit Schwermetallen wie Nickel, Kobalt, Chrom, Blei, Kupfer, Titanium, Eisen und Quecksilber sowie Kohlenstoff verunreinigt sind, was wiederum eine wesentliche Rolle bei der Entwicklung von Kontaktallergien spielen kann [10].

Henna selbst besitzt kaum sensibilisierende Wirkungen und kann allenfalls Irritationen bis hin zu einer Kontakturtikaria auslösen. Insbesondere PPD gilt als die Substanz in Henna-TattooMixturen, die dann allergische Reaktionen wie ein kontaktallergisches Ekzem, granulomatöse Typ IV-Reaktionen, prolongierte lichenoide Reaktionen bis hin zu vesikulösen, EEM-artigen Reaktionen oder Angioödemen auslösen kann [14-16]. Das Sensibilisierungspotenzial von PPD ist so hoch, dass es aufgrund des intensiven Hautkontaktes bei Henna-Tattoos zu einer Sensibilisierung mit konsekutiver allergischer Hautreaktion ohne Vorsensibilisierung kommen kann $[17,18]$. Ferner gilt PPD als Indikator für eine Gruppenallergie gegen Para-Stoffe, da eine chemische Strukturähnlichkeit zu Kreuzallergien bei PPD-Sensibilisierungen zu anderen Parastoffen wie bspw. Dispersorange 1 und 3, Dispersrot 1 und 17, p-Aminoazobenzol, Bismark Brown R und wie bei unserer Patientin p-Toluylendiamin führen kann [11 13,19]. Weiter sind Kreuzallergien mit Medikamenten wie Sulfonamiden und Sulfonylharnstoffen, Lokalanästhetika wie Benzocain und Procain sowie Sonnenschutzmitteln (häufiger Inhaltsstoff: p-Aminobenzoesäure) möglich [20]. Hier ist eine Sensibilisierung über jeweils eine Substanz und Auslösung über eine andere der o.g. möglich, was bei der Anamnese mitbedacht werden muss. Im geschilderten Fall berichtet die Patientin zwar, dass sie gelegentlich die Haare blond färbe, dies allerdings vor und nach dem ersten Henna-Tattoo jeweils mit der gleichen Farbe ohne Beschwerden, sodass eine Sensibilisierung durch Haarefärben unwahrscheinlich ist. Dennoch empfahlen wir der Patientin aufgrund der neu diagnostizierten Typ IV-Allergie auf PPD und pToluylendiamin, die Haare wegen möglicher Kreuzreaktionen nicht mehr zu färben bzw. den Kontakt zu Parastoffen strikt zu meiden. Dies ist allerdings gar nicht so einfach, da PPD - außer 
in Henna-Tattoos und Haarfarben (in der EU in einer Konzentration bis zu 6\%) - auch als Bestandteil von Farbstoffen für dunkle Lederwaren und Textilien eingesetzt wird [23].

Die positive Reaktion im Epikutantest auf Monoethanolamin stellt derzeit allenfalls eine stumme Typ IV-Sensibilisierung ohne klinische Relevanz dar, ein Zusammenhang zur Tattoo-Applikation scheint eher unwahrscheinlich. Auch beruflich hat die junge Arzthelferin keinen Kontakt zu dieser bei Metallarbeitern häufig vorkommenden Substanz. Studien belegen, dass eine positive Reaktion im Epikutantest oftmals irritativ zu bewerten ist bzw. oft eine falsch positive Reaktion vorliegt. Da Monoethanolamin allerdings Haarfärbemitteln und Haartönungen beigemengt sein kann, könnte die Patientin allenfalls hierüber eine Sensibilisierung erworben haben $[24,25]$.

Aus dem geschilderten Fall wird deutlich, dass die Anwendung von Henna-Tattoos ein ernst zu nehmendes Problem darstellt. Es muss weiter daran gearbeitet werden, die Beimengung von PPD nicht nur in Deutschland, sondern auch in anderen Ländern zu verhindern. Henna-Tattoos erfreuen sich insbesondere bei Jugendlichen einer immer größeren Beliebtheit, da sie als vorübergehendes Tattoo eingesetzt werden können, schmerzlos applizierbar und sehr preiswert sind. Daher werden sie leider immer noch allzu oft verharmlost $[21,22]$. Es muss durch weitere Aufklärungs- und Öffentlichkeitsarbeit vor der Henna-Tätowierung gewarnt und auf die oft damit einhergehende PPD-Sensibilisierung und alle daraus resultierenden Folgeprobleme deutlich hingewiesen werden.

\section{Abstract}

\section{Type IV Allergic Reaction to P-Phenylendiamin Results in Catpaw Like Eczema Following Henna Tattoo \\ $\nabla$}

We report on a young female patient with allergic contact dermatitis bearing traces of a cat beginning 3 to 5 days after the application of a henna-tattoo. In patch testing we found a type IVallergy to p-phenylendiamin that was responsible for the reaction.

Under topical therapy with class III-cortiocosteroides the contact dermatitis healed quickly. Cross-sensitization to other contact allergens such as in our case to para-toluylendiamin may occur. PPD is still an important allergen with high sensitation potential in business and private life.

\section{Literatur}

1 BfR warnt erneut vor Henna-Tattoos; Die Inhaltsstoffe können für allergische Reaktionen den Weg bereiten; (14/2007, 18.7.2007 und 10.4.2004)

2 Valsecchi R, Leghissa P, Di Landro A et al. Persistant leukoderma after henna tattoo. Contact Dermatitis 2007; 56: 108 -109

3 Lestringant GG, Bener A, Frossard P. Cutaneous reactions to henna and associated additives. Br J Dermatol 1999; 141: 598 -600
4 Schnuch A, Aberer W, Agathos M et al. Leitlinien der Deutschen Dermatologischen Gesellschaft (DDG) zur Durchführung des Epikutantests mit Kontaktallergenen. Hautarzt 2001; 52: 864-866

5 Dreborg S. EAACI: skin tests for diagnosis of IgE-mediated allergy. Allergy 1989; 44 (Suppl 10): 31 - 37

6 Ho SG, White IR, Rycroft RJ, Mc FaddenJP. A new approach to patch testing patients with para-phenylenediamine allergy secondary to temporary black henna tattoos. Contact Dermatitis 2004; 51: 213-214

7 Farrow C. Hair dye and henna tattoo exposure. Emergency Nurse 2002; 10: $19-23$

8 Neri MD, Guareschi E, Savoia F. Childhood allergic contact dermatitis form henna tattoo. Pediatric Dermatology 2002; 19: 503-505

9 Hausen BM, Kaatz M, Jappe $U$ et al. Henna/p-Phneylendiaminkontaktallergie; Folgenschwere Dermatose nach Henna-Tätowierungen. Deutsches Ärzteblatt 2001; 98: A1822 - A1825

10 Kang IJ, Lee MH. Quantification of para-phenylendiamine and heavy metals in henna dye. Contact Dermatitis 2006; 55: 26-29

11 Ho SG, White IR, Rycroft RJ, McFadden JP. A new approach to patch testing patients with para-phenylenediamine allergy secondary to temporary black henna tattoos. Contact Dermatitis 2004; 51: 213-214

12 Kligman A. The identification of contact allergens by human assay. J Invest Dermatol 1966; 47: 393-402

13 Diepgen TL. Para-Phenylendiamin - wird eine häufige und wichtige Kontaktsensibilisierung in Deutschland übersehen? Dermatologie in Beruf und Umwelt 2009; 57: 92 -93

14 Schulz J, Lonsdorf A, Jappe U, Hartschuh W. Disseminierte granulomatöse Typ IV Reaktion nach Tätowierung. Hautarzt 2008; 59: 567-570

15 Schultz E, Mahler V. Prolonged lichenoid reaction and cross sensitivityx to para substituted amino compounds due to temporary henna tattoo. Int J Dermatol 2002; 41: $301-303$

16 Sidewell R, Francis ND, Basarab T, Morar N. Vesicular erythema multiforme like reaction to para phenylendiamine in a henna tattoo. Paediatric Dermatology 2008; 25/2: 201 - 204

17 Lim SPR, Prais L, Foulds IS. Henna tattoos for children: a potential source of para-phenylenediamine and thiuram sensitization. Br J Dermatol 2004; 151: 1271

18 Cesko E, Dissemond J. Erst cooler Armschmuck, dann heißer Hautbefund. MMV-Fortschritte der Medizin 2005; 147: 61

19 Plaza T, Stevens B, von den Driesch P. Allergisches Kontaktekzem mit Gesichtsödem auf Haartönung (Color Touch ${ }^{\circledR}$ ) als Differenzialdiagnose des akuten Angioödems. Notfall \& Hausarztmedizin 2007; 33: 156 158

20 Boschnakow A, Treudler R, Lieps D, Steinhoff M, Orfanos CE. Temporäre Tätowierung mit Henna induziert Kontaktallergie auf Textilfarbstoffe. JDDG 2003; 1: $962-964$

21 Marcoux D, Couture-Trudel PM, Riboulet-Delmas G, Sasseville D. Sensitation to para-phenylendiamin from an streetside temporary tattoo. Paediatric Dermatology 2002; 19/6: 498 -502

22 Garijo-Gonzalez MA, Fernandez-Duran DA, Perez-Calderon R, SanchezCarvajal J. Allergic contact dermatitis due to a temporary henna tattoo, a hair dye und a marker pen. J Investig Allergol Clin Immunol 2008; 18: $226-227$

23 Onder $M$. Temporary holiday tattoos may cuase lifelong allergic contact dermatitis when henna is mixed with PPD. J Cosm Dermatol 2004; 2: $126-130$

24 Geier J, Lessmann H, Schnuch A, Uter W. Diagnostic quality of the patch test preparation monoethanolamine $2 \%$ pet. Contact Dermatitis 2005; 52: $171-173$

25 Lessmann H, Uter W, Schnuch A, Geier J. Skin sensitizing of ethanolamines mono., di., an triethanolamine. Data analysis of a multicenter surveillance network (IVDK) and review of literature. Contact Dermatitis 2009; 60: $243-255$ 